\title{
Die Metaphorik Darwins und Freuds
}

\section{Überlegungen zu ihrer möglichen Wirkung [1979/1986]}

Die Ordnung der Natur hat in unserer Geschichte die Bedeutung eines Spiegels, in dem der Mensch eine höhere Weisheit und seinen Standort in der Rangfolge der Naturwesen zu erkennen glaubt und an dem er sein soziales Verhalten ausrichtet und rechtfertigt. Als Walther von der Vogelweide während des Interregnums von 1198 durch sein Lied Ich hôrte ein Wazzer diezen dazu aufforderte, Philipp von Schwaben die Kaiserkrone aufzusetzen, berief er sich auf die allgemeine Schöpfungsordnung: selbst die Fliege habe ihren König, und das Ansehen des Reichs gehe in Chaos unter.

Eine orientierende Funktion behielt die Auffassung von der Natur auch, als ihre maßgebliche Auslegung sich von dem theologisch gebundenen Denken löste und in die Hand der sich verselbständigenden, sammelnden und rubrizierenden, experimentierenden und erklärenden Naturwissenschaft überging.

Dabei gehen zwei Vorgänge parallel: die Naturwissenschaften erschließen ein in den verschiedensten Richtungen sich ausweitendes Wissens- und Erfahrungsgebiet und erreichen zunehmend das allgemeine Lesepublikum der Gebildeten; Ende des 18. Jahrhunderts interessieren sich Adel und Bürgertum für naturwissenschaftliche Fragen. Damit wandelt sich die Rolle des Naturwissenschaftlers; im Zuge der Säkularisierung übernimmt er zunehmend Aufgaben, oder lässt sie sich aufdrängen, die vorher im Rahmen von Theologie und Kirche versehen worden waren: die Funktion der allgemeinen Weltinterpretation. Mit dem Aufstieg der Naturwissenschaften beginnt auch die Zeit des naturwissenschaftlichen Schriftstellers.

Ein Ausdruck dieser Entwicklung ist, daß die Wissenschaftssprache von dem gelehrten Latein zur Volkssprache übergeht. Dieser Übergang vollzieht sich in Deutschland in der zweiten Hälfte des 18. Jahrhunderts. Latein verschwindet um diese Zeit zunehmend als Sprache der deutschen Naturwissenschaft, und lateinisch geschriebene Werke ausländischer Gelehrter werden ins Deutsche übersetzt.

\footnotetext{
Anmerkung: Diese Überlegungen wurden am 27.1.1982 im Rahmen eines Colloquiumvortrags über „Die Sprache der Naturwissenschaft - Aspekte ihrer Geschichte und ihres Einflusses auf die Gemeinsprache“ im Wissenschaftskolleg zu Berlin zur Diskussion gestellt; es folgte eine kritische Replik von Wolf Lepenies in einer internen Gesprächsrunde am Kolleg und darauf die vorliegende Fassung als Vortrag am Institut für Geschichte der Medizin der FU Berlin. Zu den Ausgangsthesen vgl. meine Freiburger Antrittsvorlesung „Zur Metaphorik der naturwissenschaftlichen Sprache dargestellt am Beispiel Goethes, Darwins und Freuds“ aus dem Jahr 1978 (in diesem Band).
} 
Der Übergang der Wissenschaftssprache vom Latein zur Gemeinsprache wurde zu Beginn des 18. Jahrhunderts von Leibniz unter dem Leitgedanken einer allgemeinen nationalen Aufklärung gefordert - ob er sich immer im Sinn der Aufklärung ausgewirkt hat, ist eine Frage. Die veränderte Situation - der Rollenwandel des wissenschaftlichen Autors und die Verbreiterung seines Publikums wirkte mehr oder weniger zurück auf den Stil und die Begrifflichkeit der Autoren selbst. Sie brachte die bewunderte Fachprosa des 19. Jahrhunderts hervor, aber wohl auch eine öffentlichkeitswirksame Ungenauigkeit des wissenschaftlichen Schriftstellers, die es erlaubte, seine Begriffsprägungen und Denkmodelle über die Grenzen des in ihr abgedeckten Fachgebietes hinaus in andere Gebiete zu übertragen.

Der Biologe Oscar Hertwig schrieb 1916, also während des Ersten Weltkriegs, im Nachwort zu seinem Buch Das Werden der Organismen:

Die Auslegung der Lehre Darwins, die mit ihren Unbestimmtheiten so vieldeutig ist, gestattete auch eine sehr vielseitige Verwendung auf anderen Gebieten des wirtschaftlichen, des sozialen und des politischen Lebens. Aus ihr konnte jeder, wie aus einem delphischen Orakelspruch, je nachdem es ihm erwünscht war, seine Nutzanwendungen auf soziale, politische, hygienische, medizinische und andere Fragen ziehen und sich zur Bekräftigung seiner Behauptungen auf die Wissenschaft der darwinistisch umgeprägten Biologie mit ihren unabänderlichen Naturgesetzen berufen. (Hertwig 1916: 710)

Hertwig spricht davon, dass bei dieser Nutzanwendung der Biologie „soziale Gefahren“ entstehen könnten.

Man glaube doch nicht, daß die menschliche Gesellschaft ein halbes Jahrhundert lang Redewendungen, wie unerbittlicher Kampf ums Dasein, Auslese des Passenden, des Nützlichen, des Zweckmäßigen, Vervollkommnung durch Zuchtwahl usw. in ihrer Übertragung auf die verschiedensten Gebiete wie tägliches Brot gebrauchen kann, ohne in der ganzen Richtung ihrer Ideenbildung tiefer und nachhaltiger beeinflußt zu werden. (Hertwig 1918: 2)

Oscar Hertwig, zu der Zeit Direktor des anatomisch-biologischen Instituts der Universität Berlin und Anhänger der Sozialdemokraten, war ein Schüler Ernst Haeckels, aber ein Abtrünniger, der sich zu einem Gegner des Darwinismus entwickelt hatte. In seinem Buch Das Werden der Organismen versucht er - dies der Untertitel - „Eine Widerlegung von Darwins Zufallstheorie“.

Er beschränkt sich darin auf eine Auseinandersetzung innerhalb der Grenzen seines Fachs. Im unmittelbaren Anschluss daran aber schreibt er eine Streitschrift 
gegen die Nutzanwendung des Darwinismus, die noch während des Krieges erschien: Zur Abwehr des ethischen, des sozialen, des politischen Darwinismus. In dieser hellsichtigen Schrift, die heute kennt, wer sich mit dem Sozialdarwinismus beschäftigt, wird dieses Schrifttum wohl zum ersten Mal ausgebreitet, in Zitaten von Nietzsche, Tille, Schallmayer, Ploetz, Ammon, Wagner, Schriften mit dem Titel Grundlinien einer Rassenhygiene (Ploetz) oder Der Krieg als schaffendes Weltprinzip (Wagner).

Die wissenschaftliche Basis des Angriffs ist Hertwigs Kritik an der Evolutionslehre Darwins und - an seiner Sprache. Die Kritik an dem Naturbild Darwins erscheint ihm deshalb als so wichtig, weil es, Auslöser und Legitimationsgrund einer sozialpolitischen Bewegung, Züge einer neuen „Naturreligion“ hat. Hertwig zieht in diesem Zusammenhang einen Vergleich mit Rousseau. „Auch hier wurden aus dem dogmatisch erschlossenen und poetisch ausgeschmückten Naturzustand der Menschen die ,unvergänglichen Menschenrechte“ hergeleitet“ (Hertwig 1918: 2). - Der Vergleich ist interessant, weil im Fall Rousseaus ja in der Tat eine anthropomorphe Naturutopie das Denkmodell für eine Sozialtheorie liefert. Ganz Ähnliches hatte Friedrich Engels an einer bekannten Stelle von Darwin und seinen Nutzanwendern gesagt:

Die ganze Darwinsche Lehre vom Kampf ums Dasein ist einfach die Übertragung der Hobbesschen Lehre vom bellum omnium contra omnes und der bürgerlich-ökonomischen von der Konkurrenz, nebst der Malthusschen Bevölkerungstheorie in die belebte Natur. Hat man dieses Kunststück fertiggebracht [...], so rücküberträgt man dieselbe Theorie aus der organischen Natur wieder in die Geschichte und behauptet nun, man habe ihre Gültigkeit als ewige Gesetze der menschlichen Gesellschaft nachgewiesen. (Brief an Lawrow, 15. Nov. 1875; zit. nach Topitsch 1962: 215)

Hertwig kritisiert nicht nur, dass „die Lehre von der natürlichen Zuchtwahl im Kampf ums Dasein“ als „Naturgesetz“ ausgegeben wird, sondern auch die Art der Begriffsbildung Darwins, seine Ausdrucksweise, und zwar im Einzelnen:

1. ihre vieldeutige Unbestimmtheit - der Ausdruck ,Kampf ums Dasein` werde derartig weit gebraucht, dass sein Inhalt ganz unbestimmt werde;

2. ihren bildlichen, metaphorischen Charakter - er sei die Ursache der Unbestimmtheit. Das gelte auch für die Begriffe der ,Konkurrenz‘ und den anthropomorphen Ausdruck ,natürliche Auslese‘. In die nüchterne sachliche Sprache der Wissenschaft werde eine ,dichterische Lizenz' hineingetragen.

3. enthalten die Begriffe Darwins nach Hertwig wissenschaftlich nicht vertretbare Werturteile, so in den ,Schlagworten“,Überleben des Passenden, des Nützlichen, des Tüchtigen, des Zweckmäßigen‘. Hertwig kritisiert also die terminologische Unschärfe der Begriffe Darwins und das Vorhandensein eines ,emotiven Konnotats‘. Die Unbestimmtheit sei die Ursache dafür, dass 
diese Begriffe so vielseitig verwendbar seien und als ,Mädchen für alles“ dienten (vgl. Hertwig 1918: 2, 10f., 13, 15, 21).

Bei ihrer Übertragung in andere Bereiche - außerhalb des Zusammenhangs, wie ihn die Theorie verlange - verlören sie noch mehr an Bestimmtheit und würden zu abgegriffenen Scheidemünzen, die zu häufigen Irrtümern Anlass gäben.

Ist die Sprache Darwins tatsächlich an seiner unglücklichen Wirkung beteiligt?

\section{II}

Darwin selbst hat seine Evolutionslehre nicht in eine Sozialtheorie umgemünzt oder doch nur in kleinen Ansätzen.

Als ein deutscher Autor, Hugo Thiel, ihm eine Abhandlung über landwirtschaftliches Genossenschaftswesen sandte, in der er das Konkurrenzprinzip allgemein auf die Beziehungen der Menschen zueinander anwandte, schrieb ihm Darwin (zit. bei Preyer 1896: 168f.): „Es ist mir früher nicht beigefallen, daß meine Ansichten auf so weit abweichende und höchst wichtige Gegenstände ausgedehnt werden könnten.“ - Das klingt ein bisschen nach understatement; soviel ist richtig, dass Darwin in diesem Punkt sehr spröde war. Seine Formeln wurden aber sehr bald von anderen wissenschaftlichen Disziplinen aufgenommen, u.a. von A. Schleicher in der Sprachwissenschaft und von Carl du Prel auf dem Gebiet der Astronomie. Vor allem wurden Darwins Begriffe schlagartig in den Bereich der Politik und Sozialtheorie übertragen, zunächst in England. Schon im Jahr des Erscheinens von Darwins Hauptwerk veröffentlichte Walter Bagehot eine Schrift über Natur und Politik, in der er das Prinzip der natürlichen Auslese auf die Beziehungen der Nationen untereinander anwandte und in Krieg und Unterdrückung ein Vehikel des Fortschritts sah. Spencer übertrug das Prinzip der ,natürlichen Auslese' und ,Erhaltung begünstigter Rassen“ auf die menschliche Gesellschaft - unter der wirksamen Formel ,survival of the fittest ${ }^{\text {‘ }}$ - deutsch ,Überleben der Tüchtigsten‘.

Es gibt Übertragungen von Darwin, die halbbewusst oder unbewusst vor sich zu gehen scheinen; in einer Art von schleichendem Übergang schließt sich an die Rede von der Pflanzen- und Tierwelt die vom Menschen an. Die Autoren springen zwischen den Sphären der Naturgeschichte und der Menschengeschichte hin und her, ohne es zu merken; ihre Brücke ist die verklammernde Sprache. Und dann gibt es die Form ausdrücklicher Übertragung, die bewusst vorgenommen wird. Auch das ist häufig der Fall, z.B. in der Natürlichen Schöpfungsgeschichte von Ernst Haeckel, dem Lehrer Hertwigs: 
Wenn die natürliche Züchtung, wie wir behaupten, die große bewirkende Ursache ist, welche die ganze wundervolle Mannichfaltigkeit des organischen Lebens auf der Erde hervorgebracht hat, so müssen auch alle die interessanten Erscheinungen des Menschenlebens aus derselben Ursache erklärbar sein. Denn der Mensch ist ja nur ein höher entwickeltes Wirbelthier, und alle Seiten des Menschenlebens finden ihre Parallelen, oder richtiger ihre niederen Entwicklungszustände, im Thierreiche vorgebildet. Die ganze Völkergeschichte oder die sogenannte ,Weltgeschichte ' muß dann durch ,natürliche Züchtung‘ erklärbar sein, muß ein physikalisch-chemischer Prozeß sein, der auf der Wechselwirkung der Anpassung und Vererbung in dem Kampfe des Menschen um's Dasein beruht. (Haeckel 1870:152)

Die Rezeption Darwins durch Haeckel bezeichnet nur einen, wenn auch einen starken Strang. Darwins Wirkung war diffus. Die Evolutionslehre war ja nicht eigentlich neu, sie ist seit dem 18. Jahrhundert, seit Kant und Goethe, in einer Reihe von Stufen greifbar. Sie stand, darauf hat Wolf Lepenies besonders hingewiesen (vgl. die Vorbemerkung), in einem intellektuellen Feld, wo Aktivitäten und Disziplinen sich auszeichneten durch eine eigentümliche Kommunikationsdichte, Unschärfe und Durchlässigkeit: auf der einen Seite der Privatgelehrte, Taubenzüchter, Schriftsteller Darwin, auf der anderen eine Sozialphilosophie und Sozialwissenschaften, die bis heute in England kaum institutionalisiert sind.

Und dann hatte Darwins Ausprägung der Evolutionslehre - ich nehme noch einmal Lepenies' Akzentsetzung auf - eine ,große Deutungsbreite'. Die Wirkung weist ein beträchtliches Spektrum auf, in den USA z.B., in England, Frankreich, Deutschland - sie reicht von der Beanspruchung Darwins für eine energische Sozialpolitik oder das laissez-faire bis zu Imperialismus und Eugenik. In den Übertragungen wird also ein beträchtlicher Interpretationsspielraum der Metaphern erkennbar. Das erledigt unsere Fragestellung aber nicht, es erschwert und verschärft sie.

Wie erklärt sich die Wirkung der Begriffe Darwins? Jost Trier (1934: 196) spricht gelegentlich von Wortfeldern „mit bewährter Kraft der WeltaufschlieBung“, die sich in bestimmten Zeiten über andere Sachbereiche analogisch ausbreiten, sich „als Mittel anbieten, auch andere Bereiche analogisch zu klären“. „Sie haben Tore zur Welt geöffnet, sie werden andere Tore öffnen“. - In der Tat: erfolgreiche wissenschaftliche Konzepte haben die Tendenz, überzugehen in Nachbarfächer und in die Umgangssprache. Ein Begriffsapparat, der sich in einem Fach bewährt hat, neigt dazu, von anderen Bereichen entliehen zu werden. Newton, Darwin, Freud sind dafür Beispiele. Etwas Sozialpsychologisches ist im Spiel. Es ist ein Vorgang der Entlehnung, innerhalb der Schichten und Gruppen einer Sprachgemeinschaft, eine Form des ,social borrowing‘: „Bei Entlehnungen kommt es vor allem aufs Prestige an“, meint Ullmann (1967: 94f., 178ff.) in seiner Semantik. Übernommene Wörter evozieren das Prestige ihres ursprünglichen Milieus. Es ist eine Form der Pestigeanleihe. 
Wenn also suggestive Vorstellungsbezirke zur Expansion ihrer Sprache neigen, so gibt es umgekehrt eine Sogwirkung, die von bestimmten Zielbereichen ausgeht. Die Attraktionskraft der Zielbereiche war vermutlich in diesem Fall entscheidend. Das von Darwin Geschilderte, Konkurrenzkampf und Selektion im Reich der Pflanzen und Tiere, konnte gerade in der Mitte des 19. Jahrhunderts als Spiegel der sozialen und politischen Realität erscheinen. Die Eignung der Hauptbegriffe Darwins, in einer Epoche wirtschaftlicher Konkurrenzkämpfe und des westlichen kolonisatorischen Imperialismus als politische und Sozialtheorie instrumentalisiert zu werden, liegt auf der Hand. Noch einmal: hatte die Sprache Darwins daran Anteil? War die Kritik Hertwigs in diesem Punkt berechtigt? Kritik an der Sprache ist oft nahezu unlösbar mit der Kritik an der Sache verquickt, ist oft nur ein Vorwand; Sprachkritiker meinen häufig in der Kritik an einem neuen Sprachgebrauch die Kritik an neuen Auffassungen und Realitäten. Gilt das auch im Fall Darwins, oder ist die Bildlichkeit, Unbestimmtheit und der implizit wertende Charakter seiner Termini, von denen Hertwig spricht, tatsächlich mitverantwortlich für den breiten Übertragungsvorgang, der unmittelbar nach Erscheinen seines Hauptwerks einsetzte?

Ist die Rezeption Darwins ein Prozess der Verdrängung, indem die schwer erträgliche revolutionäre Entdeckung einer Entwicklungsmechanik umgeleitet wurde in eine Auffächerung von Missverständnissen, die rückwärtsblickende naturwissenschaftliche Tatsachenbeschreibung umdeutend in ein sozialpolitisches Programm?

Ich möchte im Folgenden versuchen, das sprachliche Problem isoliert $\mathrm{zu}$ betrachten - die Richtung unserer Überlegungen einzuengen auf die Frage nach der Metaphorik Darwins als Ursache seiner fachübergreifenden Wirkung.

\section{III}

Die Evolutionslehre Darwins arbeitet mit einigen wenigen erklärenden Begriffen oder Formeln, die erlauben, ein großes Feld naturwissenschaftlicher Daten unter dem Gesichtspunkt der Entwicklung und des Fortschritts in der Natur zu überblicken. Der Titel seines Hauptwerks, das 1859 erschien, enthält diese Begriffe oder ist eine solche Formel: On the Origin of the Species by Means of Natural Selection, or The Preservation of Favoured Races in the Struggle for Life. Eine deutsche Übersetzung von Bronn erschien schon 1860 unter dem Titel: Über die Entstehung der Arten im Tier- und Pflanzenreich durch natürliche Züchtung, oder Erhaltung der vervollkommeten Rassen im Kampf ums Dasein.

Die wichtigsten Begriffe, ,Struggle for Life“ und ,Natural Selection', sind dem Bereich menschlicher Beziehungen und Tätigkeiten entlehnt; darauf macht Dar- 
win selbst aufmerksam. Der Begriff ,Natural Selection“ wird von ihm in Analogie zu dem menschlichen Züchter gebildet - die Natur züchtet, indem sie unbewusst die am besten ausgestatteten und anpassungsfähigsten Arten auswählt und überleben lässt, so wie der Mensch durch bewusste Auslese züchtet.

Der Begriff ,Struggle for Life“ (der die treibende Kraft in diesem Auslesevorgang bezeichnet) entstand unter dem Eindruck der Bevölkerungslehre von Malthus, die besagt, dass das gesetzmäßige Missverhältnis zwischen dem Wachstum der Nahrung (in arithmetischem Verhältnis) und der Übervermehrung der Bevölkerung (in geometrischem Verhältnis) unvermeidlich zu Kriegen und einer Abwärtsentwicklung der menschlichen Gesellschaft führen müsse. „Es ist die Lehre von Malthus, in verstärkter Kraft übertragen auf das gesamte Tier- und Pflanzenreich“, schreibt Darwin (1863: 75, vgl. 15).

Im Gegensatz zu dieser pessimistischen Bevölkerungslehre sah Darwin allerdings den Konkurrenzdruck in der Natur als Vehikel des Fortschritts; man hat öfters auf die Parallele zum Wirtschaftsliberalismus der Zeit Darwins hingewiesen; für diesen Herkunftsbereich des Begriffs spricht, dass Darwin sich sehr oft ökonomischer Begriffe in seinem Werk bedient.

Die Hauptbegriffe Darwins sind also anthropomorphe bzw. - ein Ausdruck von Topitsch - soziomorphe Denkmodelle (vgl. Peters 1960: 22-37, bes. 30ff.; Topitsch 1962: bes. 213ff.; Fellmann 1977: 285-297); ihr metaphorischer und deshalb nur teilweise adäquater Charakter wird von Darwin selbst anerkannt. So schreibt er z.B. über den Begriff ,Struggle for Life‘ (in der Übersetzung Bronns): „Ich will voraussenden, daß ich den Ausdruck ,Ringen ums Dasein“ in einem weiten und metaphorischen Sinne gebrauche, in sich begreifend die Abhängigkeit der Wesen voneinander und, was wichtiger ist, nicht allein das Leben des Individuums, sondern auch die Sicherung seiner Nachkommenschaft.“ Er nennt verschiedene Beispiele - das Kämpfen zweier Hunde um Nahrung, die Abhängigkeit einer Wüstenpflanze von der Feuchtigkeit, der Mistel von dem Baum, auf dem sie schmarotzt - und schreibt: „In diesen mancherlei Bedeutungen, welche ineinander übergehen, gebrauche ich der Bequemlichkeit halber den Ausdruck ,ums Dasein Ringen““ (Darwin 1863: 76).

Der Begriff hebt also an einem komplexen und umfangreichen Vorstellungsbezirk ein gemeinsames Merkmal hervor, etikettiert diesen Vorstellungsbezirk in zuspitzender Weise und umfasst ihn so in einem Gesamtüberblick. Verbindet sich diese ,pointierende Abstraktion' mit einem unscharfen Bild- und einem starken Gefühlsgehalt, so gerät er in die Nähe dessen, was man als Definition des Schlagworts bezeichnen kann.

Tendieren die Begriffe schon bei Darwin dazu, sich als handliche Formeln von der Basis der Materialausbreitung und des Darstellungszusammenhangs zu lösen? 
Darwin ist sich der Richtigkeit seiner Termini nicht ganz sicher gewesen. „Kampf“ z.B. ist ein aktives und doppelseitiges Vorgehen. Bei vielen Lebewesen und den Pflanzen kann man aber höchstens von ,Mitbewerbung‘ um die Lebensbedingungen sprechen, von Widerstandsfähigkeit gegen widrige Bedingungen. Darwin schreibt am 30. März 1869 an W. Preyer: „Hinsichtlich des Ausdruckes struggle for existence habe ich stets einige Zweifel empfunden, war aber nicht imstande, eine bestimmte Linie zwischen den beiden darin einbegriffenen Ideen zu ziehen“. 1

Wenn ein Begriff der Alltagssprache (wie z.B. ,Kraft‘ oder ,Bewegung‘ in der Physik) terminologisiert wird, erfährt er eine Spezialisierung der Bedeutung durch Verengung seines Verwendungsumfangs und die normierte Bestimmung seines Inhalts. Der Assoziationshof von Gefühlen und Wertungen, das Konnotat, wird weitgehend ausgeblendet.

Das ist bei einem Begriff wie ,Struggle for Life‘ nur bedingt möglich. Es handelt sich ja um einen satzmäßigen Ausdruck, ein Prädikat, das, wenn es auf einen neuen Inhalt bezogen wird, immer wieder durchschlagen wird. Der Ausdruck behält von dem Bereich her, aus dem er entlehnt worden ist, ein Anschauungsmoment und insofern etwas Zuspitzendes. Er blendet manches aus. Er behält schließlich - Anschauung und Gefühle sind in der Sprache eng verknüpft, wer polemisch wird, neigt z.B. zur Anschaulichkeit - ein Konnotat von Gefühlen und Wertungen.

Wenn ein Begriff der Wissenschaftssprache in die Populärwissenschaft und in die Umgangssprache übergeht, geschieht das Gegenteil einer Spezialisierung:

Der Begriff erfährt eine Bedeutungserweiterung, eine Erweiterung des Verwendungsumfangs und eine Verallgemeinerung und Verarmung des Inhalts. Er wird vielseitig verwendet und verliert den spezifischen, klaren begrifflichen Inhalt. Er nimmt Gefühle und Wertungen der Umgangssprache in sich auf.

Das ist beim Wiedereintritt des Begriffs ,Struggle for Life“ in die allgemeine Bildungs- und Umgangssprache verstärkt der Fall. - Der Ausdruck bringt überdies das Prestige, das Konnotat der Wissenschaftlichkeit in die Umgangssprache; er hat die Würde eines allgemeinen Lebensgesetzes angenommen. Da es sich um einen satzmäßigen, bildhaften Ausdruck handelt, wird er sehr leicht etymologisch aufgefasst, d.h. sein Inhalt wird aus dem Wort, aus den Wortbestandteilen erschlossen.

Beides zusammen, die Sanktionierung als allgemeines Lebensgesetz und die auf ein Anschauungsmoment begrenzte pointierende Abstraktion des Begriffs,

1 Zit. bei Krause 1885: 85. Zur Frage der „beiden darin einbegriffenen Ideen“ vgl. Wigand 18741877, Bd. 3: $85 \mathrm{ff}$. 
führt dazu, dass er - als begrifflicher Erfassungsversuch der im Vergleich zum Pflanzen- und Tierreich ungleich komplexeren menschlichen Gesellschaft - in diesem Bereich noch weit mehr Phänomene ausblenden muss als das bei der ersten Übertragung ins biologische Feld der Fall war.

Vergleichbares lässt sich auch von dem Begriff ,Natural Selection“ sagen. Der Begriff erfasst einen Vorgang, die Auslese der bestangepassten Individuen; der Ausdruck personifiziert allerdings diesen Prozess. Auch er ist ein uneigentlicher, nicht ganz adäquater Ausdruck. Nimmt man ihn wörtlich, schreibt er der Natur Bewusstsein und Wahl zu, verbindet also, wie das Wort ,Wahlverwandtschaften', semantisch unverträgliche Bestandteile.

„Man kann sagen“ - in einer Überarbeitung der Entstehung der Arten hieß es dann - „man kann figürlich sagen, die natürliche Zuchtwahl sei täglich und stündlich durch die ganze Welt beschäftigt, eine jede, auch die geringste Abänderung zu prüfen, sie zu verwerfen, wenn sie schlecht, und sie zu erhalten und zu vermehren, wenn sie gut ist. Still und unmerkbar ist sie überall und allezeit, wo sich die Gelegenheit darbietet, mit der Vervollkommnung eines jeden organischen Wesens in Bezug auf dessen organische und unorganische Lebensbedingungen beschäftigt.“ (Darwin 1863: 101f.)

Was die Frage der Adäquatheit des Terminus ,Natural Selection“ betrifft, so waren Darwins Zweifel noch stärker als im Fall von ,Struggle for Life‘. - Am 14. Februar 1860 schreibt er noch an seinen deutschen Übersetzer H.G. Bronn, der Ausdruck habe den Vorzug, nicht sofort einzuleuchten:

Several scientific men have thought the term 'Natural Selection' is good, because its meaning is not obvious, and each man could not put on it his own interpretation, and because it at once connects variation under domestication and nature. (Darwin 1959, Bd. II: 72f.)

Aber schon am 6. Juni 1860 schreibt Darwin verzweifelt an Lyell:

I am beginning to despair of ever making the majority understand my notions. [...] I must be a very bad explainer. I hope to Heaven that you will succeed better. Several reviews and several letters have shown me too clearly how little I am understood. I suppose ,natural selection' was a bad term; but to change it now, I think, would make confusion worse confounded, nor can I think of a better; ,Natural Preservation' would not imply a preservation of particular varieties, and would seem a truism, and would not bring man's and nature's selection under one point of view. I can only hope by reiterated explanations finally to make the matter clearer. (Darwin 1959, Bd. II: 111)

Was wäre geworden, wenn Darwin ,Natural Preservation“ gesagt hätte? ,Preservation‘ - ,Erhaltung' bezeichnet besser den passivischen Aspekt des Vorgangs, dem Ausdruck fehlt der eine aktiv handelnde Instanz suggerierende Bedeutungs- 
anteil, er enthält im Gegensatz zu ,Selection‘ kaum ein Moment der Wertung. Freilich: er kann als ,Binsenwahrheit' erscheinen (,would seem a truism') und bringt nicht menschliche und natürliche Züchtung unter einen Gesichtspunkt... An solchen Stellen, beim stillen Bau der Begriffe, wird Geschichte initiiert, werden auf lange Zeit Weichen gestellt und Denkbahnen vordefiniert. Am 28. September 1860 schreibt Darwin an Lyell: „Talking of ,natural selection“: if I had to commence de novo, I would have used ,natural preservation'“" (Darwin 1959, Bd. II: 138f.) - Am Ende war es ein grammatisches Problem, weshalb Darwin Alternativen verwarf. - Am 2. Juli 1866 schrieb ihm Wallace, der bescheidene Mitentdecker: „The term ,survival of the fittest“ is the plain expression of the fact; ,natural selection' is a metaphorical expression of it, and to a certain degree indirect and incorrect, since [...] Nature [...] does not so much select special varieties as exterminate the most unfavourable ones." (Zit. Darwin 1959, Bd. II: 229, Anm.) Der Ausdruck habe eigentlich zwei Bedeutungen, er bezeichne das Faktum der Auslese und die durch sie bewirkte Veränderung. - Darwin antwortet am 5. Juli, Spencers Ausdruck ,survival of the fittest' sei in der Tat exzellent, aber er könne kein Verb regieren. „It is, however, a great objection to this term that it cannot be used as a substantive governing a verb; and that this is a real objection I infer from H. Spencer continually using the words, natural selection.“ (Darwin 1959, Bd. II: 230) - Es ist richtig: das Substantiv ,survival“ wie auch ,preservation“ lassen sich nicht mit einem Tätigkeitswort verbinden, sind als tätige Subjekte nicht vorstellbar. Und Darwin wollte, wie er wiederholt, natürliche und künstliche Züchtung in Verbindung bringen! - Dennoch wirkt der Brief sehr beunruhigt; Darwin empfindet diese Fragen als brisant; er erwägt, ob Spencers Terminus den seinen ersetzen könne, tröstet sich, auch Malthus sei missverstanden worden. Er zweifelt, ob der inzwischen verbreitete und eingebürgerte Terminus ,Natural Selection“ aufgegeben werden könne, ,and with all its faults I should be sorry the attempt made“ (Darwin 1959, Bd. II: 230).

Öffentlich freilich, in der zweiten oder dritten Auflage der Origin of the species, hatte Darwin sich schon vorher recht massiv der Kritik an seinem Terminus entgegengestellt (ich benutze die Übersetzung von Bronn, 1863): Mehrere Schriftsteller hätten den Ausdruck missverstanden oder unpassend gefunden.

Es unterliegt allerdings keinem Zweifel, daß buchstäblich genommen ,Natural Selection` ein falscher Ausdruck ist; wer hat aber je den Chemiker getadelt, wenn er von einer Wahlverwandtschaft unter seinen chemischen Elementen gesprochen? Und doch kann man nicht sagen, daß eine Säure sich die Basis auswähle, mit der sie sich vorzugsweise verbinden wolle. Man hat gesagt, ich spreche von ,Natural Selection ‘ wie von einer thätigen Macht oder Gottheit; wer aber erhebt gegen andere einen Einwand, wenn sie von der Anziehung reden, welche die Bewegung der Planeten regelt? Jedermann weiß, was damit gemeint, und ist an solche bildliche Ausdrücke gewöhnt; sie sind ihrer Kürze wegen nothwendig. Ebenso 
schwer ist es, eine Personifizierung der Natur zu vermeiden; und doch verstehe ich unter Natur blos die vereinte Thätigkeit und Leistung der mancherlei Naturgesetze. Bei ein bis'chen Bekanntschaft mit der Sache sind solche oberflächlichen Einwände bald vergessen. (Darwin 1963: 94)

Auch für den Linguisten würde gelten: bei Bekanntschaft mit der Sache definiert sich der Inhalt eines Begriffs von den Sachzusammenhängen her. Die motivierte Bildung wird dann nicht mehr aus den Bedeutungen ihrer Bestandteile erschlossen, sie verliert ihre Durchsichtigkeit zugunsten der in ihr intendierten Sache. Die Begriffe ,Struggle for Life' und ,Natural Selection' haben sich - darauf weisen Peters und Topitsch hin (Peters 1960: 33; vgl. auch Peters 1972: 347) - in der Evolutionslehre von ihrem ursprünglichen Bildgehalt abgelöst und verselbständigt.

Aber die Begriffe Darwins sind mehrschichtig. Sie haben eine Schicht, durch die sie mit der Alltagserfahrung, der Ökonomie und Ideologie der Epoche verknüpft sind und eine andere, die einem ausgebreiteten Tatsachenmaterial eine wissenschaftlich exakte, logisch evidente Deutung gibt. Die Wirkung war entsprechend mehrschichtig. Die Sachsteuerung des Verstehens funktioniert immer nur, solange und soweit die Sache bekannt ist, und auch dann nur halb. Der Sozialdarwinismus war nicht nur eine Sache des breiten, unwissenden Publikums, was die Darstellungen von Peters und Topitsch überbetonen, sondern durchaus auch der Fachbiologie. Allerdings, je weniger die Sache bekannt ist, um so mehr gewinnt ein wortgesteuertes Verstehen die Überhand. Es wird besonders durch metaphorische Termini begünstigt. Sie knüpfen ja an die bekannten Vorstellungen an und enthalten damit einen Hinweis auf die gemeinten begrifflichen Inhalte, man versteht sie halbwegs und wird sehr leicht $\mathrm{zu}$ der vorschnellen Meinung verführt, sie ganz zu verstehen, die Sache in dem Wort zu haben. Die breite und verzerrende Wirkung Darwins hängt, glaube ich, unmittelbar mit seiner Begriffsbildung zusammen.

Das ist nicht so gemeint, als gebe es einen Sprachdeterminismus, als sei der Mensch grundsätzlich der Sprache unterlegen und werde von ihr beherrscht. Wie man zwischen Sachsteuerung und Wortsteuerung unterscheiden sollte, so auch zwischen einer prinzipiellen und einer faktischen Determinierung durch Sprache. Prinzipiell ist jeder einzelne sprachliche Ausdruck hintergehbar; man kann ihn in Frage stellen, ihn sich übersetzen und auffächern in eine Reihe von aspektuellen Umformulierungen, man kann sich den Sachen neu gegenüberstellen, sie neu durchdenken und sich dabei bewusst halten, dass zwischen der Sprache und der Sache eine grundsätzliche Kluft besteht und der einzelne sprachliche Ausdruck nur einen Erfassungsversuch darstellt, der seinen Gegenstand nie ganz erreicht.

Aber faktisch lassen wir uns fast ständig von der Sprache beherrschen, erliegen der Autorität von Sprachbenutzern, aus Unfreiheit, Angst, Bequemlich- 
keit, folgen der Suggestivkraft schlagender anschaulicher Formeln, ohne ihren Ideologiegehalt zu bemerken und ihren Realitätsgehalt zu prüfen. Die magische Wiederholung und die Schallverstärkung durch die Medien tun das Ihre, sorgen dafür, dass auch wissenschaftliche und halbwissenschaftliche Termini in dieser Weise wirken können; die Formeln Darwins sind dafür ein frühes bemerkenswertes Beispiel. Man denkt, glaube ich, in der Regel wortgesteuert - besonders in der Universität. Die Sprache ist die Bedingung der Möglichkeit dafür, dass wir permanent in Wahnsystemen leben.

\section{IV}

Das spezielle Problem, mit dem wir uns beschäftigen, die Ablösung der Darwin'schen Formeln von ihrem Sachzusammenhang, wird erkennbarer und auch verschärft bei ihrer deutschen Übersetzung. ${ }^{2}$ Darwin gebraucht in der Regel ,struggle for life' oder ,struggle for existence und unterscheidet davon ,fight‘ oder ,war‘ oder ,battle‘. Der erste deutsche Übersetzer, Bronn, der die ersten vier Auflagen betreute (1863-69), gebraucht nebeneinander ,Ringen ums Dasein“ und ,Kampf ums Dasein‘. Der spätere Bearbeiter dieser Übersetzungen, Victor Carus, gebraucht häufiger ,Kampf ums Dasein‘, den Begriff, der dann fest wurde. Seine beiden Bestandteile erfahren eine Bedeutungsverschiebung. ,Struggle“ ist vieldeutig, es hat nach Roget's Thesaurus of English Words and Phrases (Erstausgabe von 1852) als Hauptbedeutungen ,exertion“ (Anstrengung, Bemühung), ,contest“ (Wettbewerb, Wettstreit) und ,contention' (eine Zwischenbedeutung zwischen Zwietracht, Zank, Streit, tiefgreifender Meinungsverschiedenheit, Gegnerschaft und aktiver Rivalität). - Die deutsche Übersetzung ,Kampf‘ verschiebt den Sinn des Wortes und macht ihn eindeutig fest als doppelseitige feindliche Auseinandersetzung. - Bei ,life‘ oder ,existence' denkt Darwin nicht nur an die Existenz des Individuums, sondern auch an die der Art durch Sicherung ihrer Nachkommenschaft. Das Wort ,Dasein“ vermittelt, zumal in Verbindung mit ,Kampf', fast nur noch die Vorstellung momentanen Überlebens. Der deutsche Ausdruck ist stark konnotathaltig, er dramatisiert. - Darwin, der ja schon mit ,Struggle for Life‘ unzufrieden war, hatte noch größere Zweifel an ,Kampf ums

2 Einige Hinweise verdanke ich einer Seminararbeit von B. Semrau: Zur Metaphorik der Begriffe „Kampf ums Dasein“ und „Natürliche Zuchtwahl“ im Werk Darwins und ihre möglichen Wirkungen. Freiburg Sommersemester 1981. 
Dasein“. „Ich vermute, daß der deutsche Ausdruck ,Kampf ums Dasein“ nicht ganz dieselbe Idee gibt“, schreibt er an Preyer (30. März 1869). ${ }^{3}$

Der Begriff ,Natural Selection“ wird von Bronn wiedergegeben mit ,natürliche Auslese‘ oder ,natürliche Züchtung‘. Mit anderen Übersetzungsvorschlägen, ,Adelung‘ oder ,Wahl der Lebensweise‘, ist Darwin, wie aus einem Brief an Bronn (14. Februar 1860; Darwin 1959, Bd. II: 73) hervorgeht, nicht einverstanden. - Bronn erwägt in einer Anmerkung (Darwin 1963: 14) auch ,Auswahl zur Züchtung‘ oder das neu zu bildende Wort ,Zuchtwahl‘. Diese Neubildung, ,Natürliche Zuchtwahl‘, wird dann bei Carus der feststehende Terminus, d.h., auch hier wird die Bedeutung verschoben, von ,Auslese' über ,Züchtung‘ zu ,Zuchtwahl‘, von einer eher passivischen Vorgangsbeschreibung zu einem eine aktiv handelnde Instanz suggerierenden Bedeutungsanteil; auch hier wird der Anthropomorphismus und das Konnotat des englischen Ausdrucks verstärkt. - Ebenso ist ,Überleben der Tüchtigsten' aktiver, anthropomorpher, wertender als Spencers ,survival of the fittest'.

Der Evolutionsgedanke war seit Ende des 18. Jahrhunderts ein allgemeineres Denkmodell, das sich zunehmend durchsetzte. Darwins Termini, die der Annahme einer Evolution der belebten Natur die wissenschaftliche Erklärung gaben, hatten schon deshalb eine gute Chance, in andere Gebiete überzugehen. Ihr soziomorpher Charakter, der sich in den deutschen Übersetzungen verstärkte, erhöhte diese Chance. Die Sprache Darwins hatte daran also Anteil; der Vorgang stellt sich mir so dar:

Die Übertragung der Begriffe aus der Biologie in den Sozialbereich war eine oft nur halb bewusste - Rückübertragung. Begriffe aus dem menschlichen Alltagsbereich wurden zuerst in den biologischen transferiert, sie füllten sich hier mit einem genauen, vielfach belegten Inhalt, wurden zum formelhaften Ausdruck eines allgemeinen kausal mechanischen Gesetzes: die Vorstellung ,Fortschritt durch Kampf ums Dasein und Zuchtwahl' konnte nun zurückübertragen werden in den menschlichen Bereich, von dem her ihr immer eine gewisse bildliche Unschärfe und Gefühlsbesetzung angehaftet hatte, und konnte sich hier im Sinn einer biologischen Terminologisierung und Sanktionierung der alltagssprachlichen Vorstellungen auswirken. Die vorherige Projektion begünstigte eine Rückprojektion. Die Übereinstimmung in der Sprache der beiden Bereiche legte eine Kongruenz in der Sache nahe. Die anthropomorphe bzw. soziomorphe Zoologie

3 Weiter heißt es in dem bei Krause 1885: 85, zitierten Brief: „Die Worte struggle for existence drücken, wie ich glaube, genau dasselbe wir Konkurrenz aus. Es ist im Englischen korrekt, zu sagen, daß zwei Menschen struggle for existence, die etwa in einer Hungersnot denselben Nahrungsmitteln nachjagen, und in gleicherweise wenn ein einzelner Mensch nach Nahrung jagt; oder hinwieder kann gesagt werden, daß ein Mensch, wenn er schiffbrüchig ist, gegen die Wellen der See: struggles for existence.“ 
schlug um in eine zoomorphe Anthropologie und Sozialtheorie; es trat jene Verführung durch die Sprache und Verwirrung der Sphären ein, von der in den Wahlverwandtschaften die Rede ist.

V

Man liest manchmal mit Verblüffung, wonach man gerade sucht. Bei der Rezeption Darwins im deutschen Sprachgebiet, und zwar bei Anhängern wie Gegnern, wird immer wieder seine Metaphorik und ihre Rückübertragung erörtert. Das gilt u. a. für Haeckel, Hellwald, Durdik, Wigand, du Prel, Kropotkin, Eckstein.

Ernst Haeckel, der Darwin für den deutschen Sprachbereich popularisierte und als allgemeingültige Weltanschauung, man kann fast sagen, institutionalisierte, schreibt in dem Werk Natürliche Schöpfungsgeschichte. - Gemeinverständliche wissenschaftliche Vorträge über die Entwicklungslehre [...], 1870: „Die Bezeichnung ,Kampf ums Dasein' ist vielleicht in mancher Beziehung nicht ganz glücklich gewählt [...]“ (Haeckel 1870: 143). In der 9. Auflage von 1898 findet sich hier der Zusatz: „Der ,Kampf um’s Dasein“ ist rasch ein Stichwort des Tages geworden. Trotzdem“ - Haeckel schreibt ,trotzdem“ - ,ist die Bezeichnung vielleicht in mancher Beziehung nicht ganz glücklich gewählt, und würde wohl schärfer gefaßt werden können als ,Mitbewerbung um die nothwendigen Existenz-Bedürfnisse“.“ Weiter unten definiert er: „Das Ringen der verschiedenen Individuen um die Erlangung der nothwendigen Existenz-Bedingungen, oder im weitesten Sinne gefaßt, die Wechsel-Beziehungen der Organismen zu ihrer gesamten Umgebung [...]“" (Haeckel 1898: 142, 147). Haeckel hält aber an dem Terminus ,Kampf ums Dasein` fest.

Albert Wigand (Der Darwinismus und die Naturforschung Newtons und $\mathrm{Cu}$ viers, 1874-77) unterzieht beide Begriffe Darwins einer eingehenden Kritik. ${ }^{4}$ Friedrich von Hellwald warnt in seiner vierbändigen Kulturgeschichte (4. Aufl.

4 Wigand 1874-1877, Bd.1. Von dem Begriff ,Natural Selection“ meint er, er habe zwischen künstlicher Züchtung und natürlicher Auslese „eine Ähnlichkeit im Wort geschaffen, welche über die Ähnlichkeit in der Sache weit hinaus geht [...]“ (92), und von dem anderen Begriff meint er: „Hätte man anstatt des zweideutigen Ausdrucks ,Kampf ums Dasein“ den Ausdruck ,Wettkampf ‘ oder ,Concurrenz' eingeführt, so wäre man nicht in Versuchung gekommen, so oft ganz verschiedene Dinge zu verwechseln. Im Gegensatz zu dem directen Kampf ist der hier in Betracht kommende wesentlich ein indirecter, und weil die kämpfenden Wesen nicht in unmittelbare Berührung kommen, ein unblutiger Kampf.“ (99f.) Vgl. auch Durdik 1906: bes. 71ff. 
1896-98) vor dem falschen Naturbegriff ebenso wie vor seiner Anwendung auf den menschlichen Bereich. ${ }^{5}$

Nachdem Darwins Bild von der durch den ,struggle for life“ beherrschten Natur zum Spiegel und Legitimationsgrund recht unsozialer Konzepte geworden war, hat der Sozialanarchist Fürst Peter Kropotkin dieses Bild der Natur selbst zurechtzurücken versucht. „In all diesen Szenen des Tierlebens, die sich vor meinen Augen abspielten, sah ich gegenseitige Hilfe und gegenseitige Unterstützung sich in einem Maße betätigen, daß ich in ihnen einen Faktor von größter Wichtigkeit für die Erhaltung des Lebens und der Species, sowie ihrer Fortentwicklung zu ahnen begann“, schreibt er im Vorwort zu seinem Buch Gegenseitige Hilfe in der Entwicklung (Kropotkin 1904: 0). Kropotkin fand diesen Gesichtspunkt schon bei Darwin angelegt und ausgesprochen, und er meint nun, Darwins Begriff vom ,Kampf ums Dasein“, der „eine außerordentliche Menge der verschiedensten Tatsachen [...] unter einen allgemeinen Begriff gestellt“ habe, habe bei seinen Nachfolgern - nicht bei ihm selbst, Darwin habe die Natur gekannt! - dazu geführt, dass der Blick auf diese Phänomene behindert worden sei. Der enge Sinn dieses metaphorischen Ausdrucks verselbständigte sich und bestimmte wie ein Okular die Sehweise und Forschungsrichtung. Eine Unmenge von Tatsachen wurde gesammelt, um das Phänomen des wirklichen Kampfes zu illustrieren. Es ging Darwins Theorie so: „Anstatt sie seinem Wirken entsprechend zu erweitern, haben sie seine Nachfolger noch enger gemacht.“ „Sie gelangten schließlich dazu, sich das Reich der Tiere als eine Welt fortwährenden Kampfes zwischen halb verhungerten Individuen vorzustellen, jedes nach des anderen Blut dürstend.“ „Sie erhoben den ,erbarmungslosen“ Kampf um persönliche Vorteile zu der Höhe eines biologischen Prinzips, dem der Mensch sich ebenfalls unterwerfen müsse, aus Gefahr, andernfalls in einer Welt, die sich auf gegenseitige Vernichtung gründete, zu unterliegen.“ Er zitiert Huxley, der die Tierwelt mit einem Gladiatorenkampf vergleicht: „Der Zuschauer braucht seinen Daumen nicht zu

5 Hellwald 1896-1898, Bd. 1: 161f.: „Man hat den Krieg als solchen zu verteidigen und in Schutz zu nehmen versucht, indem man ihn dem Kampf ums Dasein gleichstellte und ebenso wie diesen als ein Naturgesetz bezeichnete, dessen Herrschaft sich der Mensch nicht nach Belieben zu entziehen vermöchte. Aber dieser Vergleich ist insofern nicht passend, als der Kampf ums Dasein durchaus nicht immer, wie der Name anzudeuten scheint, ein gegenseitiger Vernichtungskrieg der einzelnen untereinander ist, sondern ebenso und weit mehr auf einem friedlichen Wettbewerb dieser einzelnen um die Existenzbedingungen beruht. Dieser friedliche Wettbewerb wird aber um so gesicherter, um so leichter möglich sein, je mehr der einzelne durch geordnete Staats- und Gesellschaftszustände dem rechtlosen Zustand der Urzeit entrückt und gegen fremde Gewalttat geschützt wird. Das Recht ist nämlich rein menschlich, hervorgewachsen aus der Gruppierung zu gesellschaftlicher Gemeinschaft, während in der Natur nur ein Recht, nämlich dasjenige des Stärkeren oder die Gewalt herrscht.“ 
senken, denn kein Pardon wird gegeben.“ Kropotkin will andererseits nicht nur Liebe, Friede und Harmonie erblicken, er will ebenso das Bild Rousseaus wie das Huxleys korrigieren (Kropotkin 1904: 1ff., 5).

Man könnte noch eine Reihe von Beispielen aufführen, z. B. den außerordentlichen Gustav Eckstein, der 1909 in der Neuen Zeit, dem Organ der Sozialdemokratie, meinte, die Zuchtwahllehre Darwins habe das Prinzip der freien Konkurrenz geadelt:

Wer sich geschämt hätte, den Jargon des Börsenblattes auf die höchsten Probleme der Menschheit anzuwenden, sprach jetzt pathetisch in der Ausdrucksweise exakter Naturforschung. Freilich, wenn man im Auge behalten hätte, daß ,Konkurrenz‘ und ,Zuchtwahl` gleichbedeutende Ausdrücke sind, die sich nur in ihrem gewohnheitsmäßigen Anwendungsbereich unterscheiden, dann wäre sofort klar gewesen, daß zum Beispiel Nietzsches ,Übermensch` und ,Herrennatur` ihr Vorbild in nichts anderem fanden, als im Börsenjobber und im ,königlichen Kaufmann`. (Eckstein 1909: 702)

Man könnte, wenn man die Geschichte der Begriffe Darwins genau nachzeichnen würde, einiges erfahren über die Ideologiegeschichte seit 1859. - Nach dem Zweiten Weltkrieg wurde der Begriff ,Kampf ums Dasein` von verschiedenen Biologen als ,unglücklich“ und ,drastisch“ bezeichnet und durch die Begriffe ,Konkurrenz‘, ,Wettbewerb‘, ,Wettstreit‘ ersetzt (vgl. Mayr 1967: 685; vgl. auch Denker 1975: 19ff., 29f.; Lorenz 1963: Beginn des 3. Kapitels).

\section{VI}

„Die Wissenschaft ist dem Licht gleich, und es ist ein allgemeines Interesse, daß es auf alle einzelnen ausgegossen sei“, schreibt Leibniz 1676 in einer Denkschrift über die Förderung der angewandten Naturwissenschaft, in der er den Übergang in eine deutsche Naturwissenschaftssprache forderte (Leibniz 1916a: 86). Dieser Übergang vom Gelehrtenlatein in die Landessprache birgt offenbar eine Dynamik, die sich nicht nur im Sinn der Aufklärung ausgewirkt hat. Wenn ich hier die Eingangsthese wiederholen darf:

Mit dem Aufstieg der Naturwissenschaften beginnt auch die Zeit des naturwissenschaftlichen Schriftstellers, beginnt ein Rollenwandel, der zurückwirkt auf den Stil der Autoren selbst, eine öffentlichkeitswirksame Ungenauigkeit des naturwissenschaftlichen Schriftstellers, die erlaubt, seine Begriffsprägungen und Denkmodelle über die Grenzen des in ihnen abgedeckten Fachgebiets hinaus in andere Gebiete zu übertragen. Im Zuge der Säkularisation übernimmt er zunehmend Aufgaben, oder lässt sie sich aufdrängen, die vorher von anderen Instanzen versehen worden waren: die Funktion der allgemeinen Weltinterpretation. 
Als ich mich im Kollegvortrag in diesem Sinne äußerte, hat mir Wolf Lepenies entgegengehalten: ob nicht die gegenläufige Tendenz stärker ins Gewicht falle. Seit dem 17. Jahrhundert, seit den Akademiegründungen, gebe es eine Verbindung von Erkenntnisanspruch und Orientierungsverzicht. Der Naturwissenschaftler ziehe sich gerade zurück aus Theologie, Rhetorik, Poesie und kopple sich ab von der Lebenspraxis. Der schriftstellernde Naturwissenschaftler erleide einen Reputationsverlust. Der umfassende Orientierungscharakter der Evolutionslehre sei eher gegen die Absicht Darwins so stark herausgestellt worden.

Ich bestreite natürlich nicht, dass es diese Tendenz seit der frühen Neuzeit, seit Descartes, gibt; sie führt bis hin zum „sprachlosen Gelehrten“ in der modernen Physik und in der neueren Medizin, von dem Eckart Matthias und Rolf Winau in unserer Diskussionsrunde sprachen (vgl. Winau 1980: 95-102). Aber es gibt auch das Umgekehrte, und zwar, keineswegs nur an den Rändern der Naturwissenschaft, in großem Maßstab. Der Übergang vom Gelehrtenlatein in die Landessprache, dieser Demokratisierungsprozess ist von einer noch gar nicht beschriebenen Bedeutung; die Verbreiterung des Lesepublikums im 18. Jahrhundert musste sich auswirken. Everett Mendelsohn (1982) hat in seinem Vortrag im Kolleg auf das Paradox aufmerksam gemacht, dass die Naturwissenschaftler in dem Augenblick, wo die Anwendungsbereiche ihrer Tätigkeit sich in alle Richtungen, Landwirtschaft, Industrie, Verkehr usw., ausweiten, am keuschesten das Postulat reiner Erkenntnis aufstellen. - Diese Antinomie sollte sich nicht in der Sprache der Naturwissenschaft niedergeschlagen haben?

Ich möchte meine These eher noch verschärfen; die Grenzen zwischen Wissenschaft und Populärwissenschaft sind seit Ende des 18. Jahrhunderts keineswegs streng. Der Prozess der Demokratisierung der Wissenschaft birgt - neben allen großartigen Horizonterweiterungen - eine gefährliche Dynamik und Dialektik. Auf Seiten der Autoren ist sie greifbar in schielenden Begriffen (die mit dem einen Auge auf die Sache und mit dem anderen auf das Publikum schauen), in den Eselsbrücken für die Allgemeinheit.

Woher kommt denn die Bedenklichkeit Darwins in sprachlichen Fragen? Warum wählte er die Begriffe, die er wählte? Schielte nicht auch er schon ein bisschen auf Wirkung?

Wolf Lepenies macht in diesem Zusammenhang darauf aufmerksam, dass er den entscheidenderen Zusammenhang in folgendem sieht: durch den spröden Verzicht eines Naturwissenschaftlers wie Darwin auf umfassende Deutung und Orientierung entsteht ein Vakuum, das eine Sogwirkung ausübt und den paradoxen Effekt einer extremen Orientierungs- und Deutungsbereitschaft bei naturwissenschaftlichen Schriftstellern im Ausstrahlungsbereich Darwins hervorruft. Das vorherrschende Phänomen in der Entwicklung der neuzeitlichen Naturwissenschaft sei jener Vorgang, den er als Ent-Moralisierung bezeichne, d.h. die Abkop- 
pelung normativer Fragestellungen von der Wissenschaftspraxis. Sie führe im 19. Jahrhundert $\mathrm{zu}$ dem Orientierungsverlust der Wissenschaften, den wir bis heute beklagen (vgl. Lepenies 1982: 67-106, bes. 97ff., 104f.; Lepenies o.J.: bes. 1, 10ff., 24).

Ich möchte aus zwei Gründen widersprechen. Der echte oder simulierte Orientierungsverzicht der Naturwissenschaftler des 19. Jahrhunderts ist nicht unbedingt deckungsgleich mit ihrer Sprache. Diese ist mehrschichtig. Sie hat, unterschwellig oder oberschwellig, immer wieder allgemein orientierende Züge, einen Weltbildcharakter. Ihr latenter Ideologiegehalt ist am leichtesten fassbar in der umgangssprachlichen Metaphorik der Naturwissenschaftler. Wir begegnen ihm deshalb vielleicht am häufigsten in der noch ganz umgangssprachlichen Biologie, weniger in der Chemie oder in der noch sehr viel weiter von der Umgangssprache entfernten Physik.

Darüber hinaus gibt es aber in den modernen Naturwissenschaften, die nach Lepenies charakterisiert sind durch die Kombination von Erklärungsanspruch und Orientierungsverzicht, sehr oft ein Übergewicht des ersten Gliedes dieses Paradoxons: einen universellen Erklärungsanspruch, die Tendenz zu einem biologistischen, chemistischen oder physikalistischen Weltbild. Diese Neigung zur Sphärenüberschreitung und ,Sphärenvermengung‘, der Übertragung von Kategorien und Denkmodellen eines naturwissenschaftlichen Faches in andere Bereiche, ihre Handhabung als universeller Schlüssel, ist eine den modernen Naturwissenschaften immanente Tendenz (vgl. den Aufsatz „Zur Metaphorik der naturwissenschaftlichen Sprache - dargestellt am Beispiel Goethes, Darwins und Freuds“ in diesem Band).

Darwin ist auf jeden Fall ein Beispiel dafür, welche Erwartung im 19. Jahrhundert an einen naturwissenschaftlichen Autor herangetragen werden kann. Sein Bild gerinnt in der Öffentlichkeit zur Zauberformel und wird auf ein Klischee gebracht. Es erliegt einer historisierenden Mythisierung.

Darwin selbst deutet gelegentlich an, dass er sich in Parallele zu Newton sieht, z. B. im Schlusssatz von Die Entstehung der Arten:

Es ist wahrlich eine großartige Ansicht, daß der Schöpfer den Keim allen Lebens, das uns umgibt, nur wenigen oder nur einer einzigen Form eingehaucht hat und daß, während unser Planet den strengsten Gesetzen der Schwerkraft folgend sich im Kreise geschwungen, aus so einfachem Anfange sich eine endlose Reihe der schönsten und wundervollsten Formen entwickelt hat und noch immer entwickelt. (Darwin 1867: 565)

Haeckel führt diesen Vergleich durch. Wir finden bei ihm eine Vorform der berühmten Äußerung Freuds über die drei Kränkungen der Menschheit: 
Durch das Weltsystem des Copernikus, welches Newton mechanisch (durch die Gesetze der Schwere und der Massenanziehung) begründete, wurde die geocentrische Weltanschauung der Menschheit umgestoßen, d.h. der Irrwahn, daß die Erde der Mittelpunkt der Welt sei, und daß die übrigen Weltkörper, Sonne, Mond und Sterne, nur dazu da seien, um sich rings um die Erde herumzudrehen. Durch die Entwicklungstheorie des Lamarck, welche Darwin mechanisch (durch die Gesetze der Vererbung und der Anpassung) begründete, wurde die anthropocentrische Weltanschauung der Menschheit umgestoßen, d.h. der lrrwahn, daß der Mensch der Mittelpunkt des Erdenlebens und die übrige irdische Natur, Thiere, Pflanzen und Anorgane, nur dazu da sei, um dem Menschen zu dienen. ${ }^{6}$

Haeckel zeichnet ihn als ,Reformator‘, fast schon im Faltenwurf des deutschen Trutz-Luthers; Darwins Bild tritt bei manchen Nachfolgern ein in den religiösen Sprachtyp.

Ein Album, das Darwin gewidmet werden sollte und das die Huldigung deutscher Anhänger versammelte, stellte auf dem Titelblatt einen hohen goldenen Bogen dar mit der Inschrift ,Dem Reformator der Naturgeschichte Charles Darwin ' und am Fuß einer ganzen Reihe allegorischer Darstellungen eine Kopie der ,Erschaffung Adams‘ von Michelangelo.

$\mathrm{Zu}$ den für das Album eingereichten Beiträgen gehörten auch die Strophen eines sonst nicht bekannten Dichters aus Pforzheim (Rade 1877: 29, 36):

\author{
Christus dort und Darwin hier \\ Leuchten als des Geistes Zier \\ Christus lehrt als Glaubensheld, \\ Daß verwandt die ganze Welt. \\ Darwin hier hat's auch erkannt \\ Daß die Körperwelt verwandt. \\ [...] \\ So wie Christus, Darwin hier \\ Leuchtet als des Geistes Zier.
}

\begin{abstract}
6 Haeckel 1902, Bd.1: 69. Vgl. Freud 1969ff., I: 283f.: „Zwei große Kränkungen ihrer naiven Eigenliebe hat die Menschheit im Laufe der Zeiten von der Wissenschaft erdulden müssen. Die erste, als sie erfuhr, daß unsere Erde nicht der Mittelpunkt des Weltalls ist, sondern ein winziges Teilchen eines in seiner Größe kaum vorstellbaren Weltsystems. Sie knüpft sich für uns an den Namen Kopernikus, obwohl schon die alexandrinische Wissenschaft ähnliches verkündet hatte. Die zweite dann, als die biologische Forschung das angebliche Schöpfungsvorrecht des Menschen zunichte machte, ihn auf die Abstammung aus dem Tierreich und die Unvertilgbarkeit seiner animalischen Natur verwies. Diese Umwertung hat sich in unseren Tagen unter dem Einfluß von Ch. Darwin, Wallace und ihren Vorgängern nicht ohne das heftigste Sträuben der Zeitgenossen vollzogen. Die dritte und empfindlichste Kränkung aber soll die menschliche Größensucht durch die heutige psychologische Forschung erfahren, welche dem Ich nachweisen will, daß es nicht einmal Herr ist im eigenen Hause, sondern auf kärgliche Nachrichten angewiesen bleibt von dem, was unbewußt in seinem Seelenleben vorgeht.“
\end{abstract}


Es ist dies nur der unfreiwillig parodistische Reflex einer religiös getönten Monumentalisierung Darwins.

Angesichts dieser Wirkung ist umso bemerkenswerter die Art, in der er sich zurückhielt. Der Mann ist als wissenschaftlicher Autor von einer geradezu begeisternden Trockenheit, Strenge und Sachlichkeit.

\section{VII}

Freud steht durch die Art seiner wissenschaftlichen Darstellung den naturwissenschaftlichen Schriften Goethes nahe und scheint sich an ihnen geschult zu haben. Das gleiche gilt in einem hohen Grad für sein Bewusstsein, dass er sich bei seiner Darstellung der tiefenpsychologischen Zusammenhänge einer bildlichen Sprache bedient und dass ein Bild nur eine Hilfsvorstellung sei, die den Gegenstand nur unvollständig erfasse. Gelegentlich schaltet er bewusst mit verschiedenen Bildvorstellungen, um, wie er sagt, „ein höchst kompliziertes und noch niemals dargestelltes Denkobjekt von verschiedenen Seiten her zu veranschaulichen“ (Freud 1940ff., I: 295). Er wählt aber bevorzugt Analogien, die den Naturwissenschaften entlehnt sind. Seine Vorstellung von der Psyche als eines Energieverteilungsapparats ist z.B. der Physik entlehnt und hat den Wert eines einen umfangreichen Bezirk erschließenden Denkmodells. Die naturwissenschaftlichen Analogien sind insgesamt mehr als bloße Metaphern - Freud verstand die Tiefenpsychologie als Teildisziplin der Naturwissenschaft und sich als Naturwissenschaftler, der nur noch nicht in der Lage sei, die psychischen Vorgänge bis in ihre physiologische, physikalisch-chemische Basis zu verfolgen. Er bevorzugt eine naturwissenschaftliche Sprache, weil er ihr den höchsten Annäherungswert an die tatsächlichen Zusammenhänge zuschreibt.

Das Wort ,Psychoanalyse` hat er der Chemie entlehnt - es verbindet, wie die Ausdrücke ,Wahlverwandtschaften“ und ,Natürliche Zuchtwahl‘, Bestandteile, die, buchstäblich genommen, semantisch unverträglichen kategorialen Bereichen angehören. Das Verb ,analysieren', als Tätigkeit des Chemikers, fordert in dem syntaktischen Plan, den es bestimmt, an der Stelle des Objekts einen unbelebten Stoff. Freud überträgt die Arbeit des Chemikers an den Stoffen in den Bereich der Beziehung zwischen Analytiker und Patient. Wenn er an einer Stelle auf das mehr als zufällige Zusammentreffen hinweist, dass Goethe „in den ,Wahlverwandtschaften' eine Idee aus dem Vorstellungskreis der Chemie auf das Liebesleben anwendete, eine Beziehung, von der der Name selbst der Psychoanalyse zeugt“ (Freud 1940ff., XIV: 549), so trifft diese Parallele nur zur Hälfte. Goethe wendete nicht eine Idee aus dem Vorstellungskreis der Chemie auf das „Liebesleben“ (ein für Goethes Roman unangemessen biologistischer Ausdruck) an - 
diese Form einer starren Projektion naturwissenschaftlicher Vorstellungen in den Bereich der menschlichen Psyche ist eher für Partien in der mechanistischen oder biologistischen Diktion Freuds charakteristisch - sondern er führte ein in der Chemie gebräuchliches ,ethisches Gleichnis‘ bewusst in den Bereich menschlicher Beziehungen zurück und füllte es durch seinen Roman mit einem differenzierten, spannungsvollen, anschaulichen und begrifflichen Gehalt. Er bereicherte den Wortschatz auf dem Gebiet menschlicher, geistiger Beziehungen um ein, wie er fand, ,geistreiches Wort'.

In einem Vortrag von 1918 erläutert Freud den Namen ,Psychoanalyse‘ und führt den Vergleich mit der Tätigkeit des Chemikers in einer Anzahl von Punkten durch (Freud 1940ff., XII: 183ff.). Der Anlass ist, dass sich eine neue Richtung der Psychotherapie zu bilden scheint, die sich ,Psychosynthese' nennt. Freud charakterisiert ihren Grundgedanken: „Wir haben den Kranken analysiert, das heißt seine Seelentätigkeit in ihre elementaren Bestandteile zerlegt, diese Triebelemente einzeln und isoliert in ihm aufgezeigt; was läge nun näher als zu fordern, daß wir ihm auch bei einer neuen und besseren Zusammensetzung derselben behilflich sein müssen?“

Freud reagiert auf dieses aus der Metapher ,Psychoanalyse“ entwickelte Programm mit auffallender Heftigkeit. Er spricht von einer „gedankenlosen Phrase“, einer „inhaltsleeren Überdehnung eines Vergleiches“ und „unberechtigter Ausbeutung einer Namengebung“ und setzt ihr folgende Sprachauffassung entgegen:

Aber ein Name ist nur eine Etikette, zur Unterscheidung von anderem, ähnlichem, angebracht, kein Programm, keine Inhaltsangabe oder Definition. Und ein Vergleich braucht das Verglichene nur an einem Punkte zu tangieren und kann sich in allen anderen weit von ihm entfernen. Das Psychische ist etwas so einzig Besonderes, daß kein vereinzelter Vergleich seine Natur wiedergeben kann.

Wenn Freud den Namen als Etikette definiert und seine nur distinktive Funktion hervorhebt, so nennt er nur einen der beiden Pole, zwischen denen sich das sprachliche Zeichen bewegen kann. Er spricht von dem Zeichenterminus, der eine nur benennende, diakritische Funktion erfüllt - wie z.B. das Abkürzungssymbol. Ein Gegenpol wäre der motivierte, logisch durchsichtige Terminus, der eine klassifizierende Funktion erfüllt, wie z.B. die logisch motivierte Zusammensetzung.

Eine zunächst durchsichtige Wortzusammensetzung kann sich nun zu dem Pol des nur etikettenhaften Zeichenterminus hinbewegen, im Sinn der von Bühler (1965: 349) beobachteten Sachsteuerung des Verstehens und der selektiven Auffassung von Metaphern, die Unzutreffendes ausblendet. Das ist auch im Fall des Namens ,Psychoanalyse` geschehen - seine ldiomatisierung lässt sich bei Freud 
belegen. ${ }^{7}$ Aber die ursprüngliche, aus den Wortbestandteilen erschließbare Bedeutung kann jederzeit wieder aktualisiert werden. Es hängt von der Art des Wortgebrauchs, von der Auffassung des Namens ab, ob ein Wort wie ,Psychoanalyse' als Zeichenterminus oder als definierender Terminus funktioniert. Freud gebraucht seine Termini meist als definierende, sprechende Ausdrücke - er hat auch das Wort ,Psychoanalyse“ in diesem Vortrag, vor dem polemischen Einschub, als definierenden Terminus verwendet. Darin fährt er jetzt fort. Der Gedanke, dass einer Psychoanalyse die Synthese folgen müsse, liegt offenbar so nahe, dass Freud selbst diesen Weg einschlägt.

Er spricht davon, „daß wir es im Seelenleben mit Strebungen zu tun haben, die einem Zwang zur Vereinheitlichung unterliegen“. Das Ich, diese große Einheit, füge sich die in der Analyse befreiten Triebregungen wieder ein. „So vollzieht sich bei dem analytisch Behandelten die Psychosynthese ohne unser Eingreifen, automatisch und unausweichbar.“ In einer Anmerkung weist Freud - als Analogon - auf die Chemie hin, und zwar auf das Gesetz der Wahlverwandtschaften: „Ereignet sich doch während der chemischen Analyse etwas ganz Ähnliches. Gleichzeitig mit den Isolierungen, die der Chemiker erzwingt, vollziehen sich von ihm ungewollt Synthesen dank der freigewordenen Affinitäten und der Wahlverwandtschaft der Stoffe.“ Freud bleibt anlässlich dieser grundsätzlichen Auseinandersetzung in den durch das chemische Denkmodell vorgeschriebenen Bahnen - er folgt anscheinend der in dem Bild liegenden Suggestion. Wieweit allerdings dabei der Sprache ein selbständiger Anteil zukommt, mag offenbleiben.

Die an sich abgelegene Stelle berührt eine Frage, die in der neueren Diskussion um Freud eine erhebliche Rolle gespielt hat: Führte der naturwissenschaftliche Ausgangspunkt zu dem unrichtigen Konzept der Psyche als eines in sich funktionierenden, selbstregulierenden Apparats? Liegt hier eine unzulässige Sphärenvermengung vor? Nach Gustav Bally wurzeln die Begriffe Freuds nicht „in der kategorialen Sphäre, in der das Phänomen zu Hause ist“. Habermas bringt das Problem auf die Formel eines „szientistischen Selbstmißverständnisses der Psychoanalyse“ und ist der Meinung, daß die naturwissenschaftliche Sprache Freuds einen nur scheinbaren Anschluss an das naturwissenschaftliche Weltbild der Jahrhundertwende herstellt (Bally 1971: 20; Habermas 1970: 308). Die Wirkung Freuds, soweit sie gerade durch dieses Weltbild gestützt wurde und dazu führte, dass seine Lehre als Weltanschauungssurrogat rezipiert wurde, hätte dann ebenso auf einem durch die Sprache begünstigten Missverständnis beruht wie

7 Vgl. den Artikel „Psychoanalyse“ von 1922, Freud 1940ff., XIII: 211. 
umgekehrt die Verweigerung der Rezeption Freuds aufgrund dieser naturwissenschaftlich-mechanistischen Sprache.

\section{VIII}

Es wäre nicht sinnvoll und könnte nur dilettantisch ausfallen, wenn ein kleiner Philologe versuchen würde, von seinem Fach her etwas zu dem Sachgebiet der Evolutionslehre oder der Psychoanalyse zu sagen, aber ich halte es für begründet, die Nahtstelle zu beobachten, an der ein wissenschaftliches Denkmodell aus einer Disziplin in andere Disziplinen oder in eine allgemeine Weltanschauung übergeht. An dieser Stelle wirkt die Sprache sich aus. Die Metapher ermöglicht das Ausgreifen oder legt es doch nahe. Eine Naturwissenschaftssprache, die sich einer anthropomorphen oder soziomorphen Metaphorik bedient, hat die Tendenz, in Anthropologie oder Sozialtheorie umzuschlagen.

Das Problem der richtigen Analogie, der Übertragung und Rückübertragung, ist seit einiger Zeit Bestandteil des heftigen Streits um Konrad Lorenz und die Tierverhaltensforschung. Lorenz, der den Kapiteln seines Buches Das sogenannte Böse mit Vorliebe Goethe-Zitate voranstellt, bedient sich bekanntlich ganz besonders häufig des Verfahrens, „etwas von dem Kreise menschlichen Wissens Entferntes durch ethische Gleichnisse näher heranzubringen“ - ,Imponiergehabe, ,Parlament der Instinkte‘, ,Triumphgeschrei“, ,Band der Freundschaft‘, ,Liebe‘, ,selbstloses Verhalten“ usw. (Lorenz 1963: passim) - und überträgt Vorstellungen aus dem Bereich des Tierverhaltens in den menschlichen zurück. Schon 1948 hatte Tinbergen auf die Gefahren hingewiesen, „,welche in dem Verfahren liegen, physiologische Erscheinungen auf einer niedrigeren Evolutionsebene, auf einem niedrigeren Niveau der neutralen Organisation und bei einfacheren Verhaltensformen als Analogien zu benutzen, um damit physiologische Theorien über Verhaltensmechanismen auf höheren und komplexeren Ebenen zu stützen“. ${ }^{8}$

Während Konrad Lorenz Darwins Begriff vom Struggle for Life kritisch betrachtet und durch eine differenzierte Analyse des Phänomens der ,Konkurrenz ersetzt, überspitzt er den in dem Ausdruck Natural Selection liegenden anthropomorphen Gehalt und erhebt die ,Natürliche Zuchtwahl` zu einer religiös empfundenen Instanz. Die Begriffe ,Mutation“ und ,Selektion“ - oder ,Erbänderung‘ und ,Zuchtwahl` - erscheinen durchgehend unter dem Titel ,Die großen Konstrukteure des Artenwandels‘, und er schreibt am Schluss seines Buches:

8 Tinbergen 1948: 121-133; hierzu und zum folgenden vgl. die aufschlussreichen Abschnitte bei Erich Fromm 1974: 20ff. 
Das volle und warme Gefühl von Liebe und Freundschaft können wir nur für Einzelmenschen empfinden, daran kann der beste Wille nichts ändern! Doch die großen Konstrukteure können es. Ich glaube, daß sie es tun werden, denn ich glaube an die Macht der menschlichen Vernunft, ich glaube an die Macht der Selektion und ich glaube, daß die Vernunft vernünftige Selektion treibt.

Die großen Konstrukteure der Natur verwandeln sich hier in die großen Konstrukteure der zukünftigen Menschheitsentwicklung, der Übergang von einer Personifikation der Natur zur menschlichen Person und einer von ihr gehandhabten Selektion ist sprachlich vollzogen. Man erinnert sich angesichts einer so bedenkenlosen, die Bereiche verwischenden Sprache an die Warnung in den Wahlverwandtschaften: „Aber der Mensch ist doch um so manche Stufe über jene Elemente erhöht und wenn er hier mit den schönen Worten [...] etwas freigebig gewesen, so tut er wohl, wieder in sich selbst zurückzukehren und den Wert solcher Ausdrücke bei diesem Anlaß recht zu bedenken.“

Glücklicherweise teilt Lorenz ebenfalls gegen Ende seines Buches die Beobachtung mit, dass der Humor stammesgeschichtlich im Wachsen begriffen sei. 